\title{
Prevalence and molecular epidemiology of Staphylococcus aureus in Swedish nursing homes - as revealed in the SHADES study $\dagger$
}

\author{
L. STARK ${ }^{1,2 *}$, M. OLOFSSON ${ }^{3,4}$, S. LÖFGREN ${ }^{1}$, S. MÖLSTAD ${ }^{5}$, \\ P.-E. LINDGREN ${ }^{1,2}$ AND A. MATUSSEK ${ }^{1}$ \\ ${ }^{1}$ Clinical Microbiology, Department of Laboratory Medicine, Ryhov County Hospital, Jönköping \\ ${ }^{2}$ Division of Medical Microbiology, Department of Clinical and Experimental Medicine, Linköping University, \\ Linköping \\ ${ }^{3}$ Ödeshög Health Care Centre, Ödeshög \\ ${ }^{4}$ Division of Community Medicine, Department of Medical and Health Sciences, Linköping University, \\ Linköping \\ ${ }^{5}$ Division of General Practice/Family Medicine, Department of Clinical Sciences in Malmö, Lund University, \\ Malmö
}

Received 3 May 2013; Final revision 29 July 2013; Accepted 29 July 2013;

first published online 20 August 2013

\section{SUMMARY}

Knowledge of carriage and population dynamics of Staphylococcus aureus is crucial for infection risk assessment and to reveal transmission patterns of strains. We report the prevalence and molecular epidemiology of $S$. aureus in elderly people $(n=290)$ living in nursing homes in three cities in the south of Sweden. The overall carriage prevalence rate was $48 \%$ when results from nares $(31 \%)$ and throat $(34 \%)$ samples were combined. Common spa types were equally distributed but a frequent type, t160, was found only in one of the regions. Carriage of different spa types was detected in $23 \%$ of individuals and antimicrobial resistance rates were higher in S. aureus isolates from those carrying more than one spa type. Five of the 21 individuals who carried different spa types were colonized simultaneously with resistant and non-resistant strains. Seventeen per cent of the individuals carried $S$. aureus of the same spa type on all occasions. Methicillin resistance was not detected. In conclusion we found a high prevalence of $S$. aureus in this elderly population with a high rate of dual colonization with different spa types.

We also found signs of institutional spread of one strain.

Key words: Colonization, molecular typing, multiclonality, nursing home, spa type.

\section{INTRODUCTION}

Molecular epidemiological studies of Staphylococcus aureus have often focused on methicillin-resistant

\footnotetext{
* Author for correspondence: Miss L. Stark, Department of Clinical Microbiology, Ryhov County Hospital, SE-551 85 Jönköping, Sweden. (Email: lisa.stark@lj.se)

$\dagger$ This study is part of the SHADES programme (the Study of Health and Drugs among the Elderly in Swedish Nursing Homes), which in general focuses on mortality, morbidity, health conditions and drug use in elderly individuals living in community dwellings in Sweden.
}

S. aureus (MRSA) in outbreak situations. Recent studies indicate that the prevalence of MRSA is low in general populations in European countries $[1,2]$. The clinical significance of methicillin-sensitive S. aureus (MSSA) is, however, also great [3, 4], and some spa types are indicated to be more common in clinical isolates compared to community isolates [5]. Differences in the distribution of MSSA and MRSA strain types have been reported [6] and show a greater diversity of spa types in MSSA [2, 5-7], compared to the often observed clonal spread of MRSA strain lineages [7, 8].

The online version of this article is published within an Open Access environment subject to the conditions of the Creative Commons Attribution licence $<$ http://creativecommons.org/licenses/by/3.0/>. 
An association of specific MSSA spa types with age and gender in the general population has been suggested [2]. Moreover, nursing homes have been implicated as reservoirs for antimicrobial-resistant organisms, including S. aureus [9]. Only a few comprehensive $S$. aureus screening studies have been performed within these settings, and most focused primarily on MRSA [10, 11].

The overall prevalence of nasal $S$. aureus colonization varies in different studies, and rates up to $55 \%$ have been reported [12]. Carriers are generally classified as persistent $(\sim 20 \%)$, intermittent $(\sim 30 \%)$ and non-carriers $(\sim 50 \%)[13,14]$. The classification of an individual as a persistent carrier is of importance, since they appear to have an increased risk of endogenous infection compared to non-carriers $[15,16]$, whereas intermittent carriers have an equally low risk as non-carriers [17]. However, it should be noted that classification of a persistent carriage state remains contentious [18].

Recent studies have shown an equal or higher colonization rate of $S$. aureus in the throat compared to nares, and there are a number of individuals exclusively colonized in the throat $[13,19,20]$. A possible role of throat carriage for $S$. aureus transmission has also been recently indicated [21], and inclusion of throat culture in screening protocols is widely considered to be necessary for the optimal recovery of S. aureus [20, 22, 23].

Knowledge of molecular epidemiology of $S$. aureus in nursing-home residents is important as this group represents a population with high comorbidity and frailty [24]. Furthermore, they have an increased frequency of hospital care with a risk of exchange of bacterial strains [9], which might have an influence on transmission patterns. The aim of this study was to determine the prevalence of $S$. aureus in Swedish nursing-home residents and investigate the molecular epidemiology of isolates in terms of the spatial and temporal spa gene type distribution and antimicrobial resistance.

\section{METHODS}

\section{Study population}

The study population ( $n=290 ; 70 \%$ women, $30 \%$ men) was recruited from individuals, between the ages of 60 and 101 (median 85) years, included in the SHADES programme [24], a longitudinal, open cohort, multipurpose study conducted in Swedish nursing homes for permanent residents, between 2008 and 2011. The nursing homes $(n=9)$ were located in three cities in the south of Sweden, Eslöv $(n=2)$ with $47 \%$ of the participants, Jönköping $(n=4)$ with $41 \%$ of the participants and Linköping $(n=3)$ with $11 \%$ of the participants. The colonization rates of $S$. aureus and antibiotic susceptibility results from the first culture have in part been reported previously [23]. Information about age, gender and length of stay were registered. The study was approved by the Regional Ethical Review Board at Linköping University (M150-07). Informed consent was obtained from all included individuals (or from persons close to them as appropriate).

\section{Sample collection and $S$. aureus isolation and cultivation}

Sampling was performed using a rayon-tipped swab (Copan Diagnostics Inc., Italy), from 1 January 2009 to 31 March 2011 and included samples $(n=1860)$ from the anterior nares, the throat, the groin and active skin lesions. If possible, samples were collected every 6 months during the study period.

The swabs were incubated for $16-20 \mathrm{~h}$ in a broth selective for $S$. aureus [13] and then $10 \mu \mathrm{l}$ broth was cultured on blood agar plates $(n=996)$, and from 1 February 2010 on BBL ${ }^{\text {TM }}$ CHROMagar ${ }^{\text {TM }}$ Staph aureus medium (Becton Dickinson, USA) $(n=864)$. Plates were incubated for 20-24 h, and $S$. aureus was confirmed by DNase activity or detection of the nuc gene [25]. Isolates were tested for antibiotic susceptibility [23] and stored at $-80^{\circ} \mathrm{C}$ in skimmed milk.

\section{spa typing}

All $S$. aureus isolates $(n=466)$ were spa typed according to Kahl et al. [26]. PCR products were sequenced at GATC Biotech (GATC Biotech AG, Germany). Ridom StaphType software (Ridom $\mathrm{GmbH}$, Germany) was used to determine the spa types [27] and BURP clustering was performed [28].

\section{Statistical analyses}

Statistical analysis ( $\chi^{2}$ test and $t$ test) was performed using the IBM SPSS Statistics version 20 statistical package (IBM Corporation, USA) and included the prevalence of colonization with $S$. aureus at the four body sites, the proportion of antibiotic-resistant 
Table 1. Detection of $\mathrm{S}$. aureus and spa-type distribution, in different body sites, at the first sampling occasion

\begin{tabular}{|c|c|c|c|c|c|c|}
\hline & \multicolumn{6}{|l|}{ Body site } \\
\hline & Nares & Throat & Groin & Skin lesion & $\begin{array}{l}\text { Nares and } \\
\text { throat }\end{array}$ & $\begin{array}{l}\text { Nares, throat } \\
\text { and groin }\end{array}$ \\
\hline Number of individuals sampled & 285 & 180 & 245 & 17 & 179 & 179 \\
\hline $\begin{array}{l}\text { Individuals from whom } S \text {. aureus } \\
\text { was isolated, } n(\%)\end{array}$ & $87(31)$ & $62(34)$ & $24(10)$ & $7(41)$ & $86(48)^{*}$ & $88(49)^{*}$ \\
\hline Number of different spa types & 59 & 43 & 15 & 7 & & \\
\hline $\begin{array}{l}\text { Most common spa types found } \\
\text { in different individuals }(\%) \$\end{array}$ & $\begin{array}{l}\text { t002 }(6 \cdot 8) \\
\text { t160 }(5 \cdot 7) \\
\text { t015 }(5 \cdot 7) \\
\text { t295 }(3 \cdot 4) \\
\text { t008 }(3 \cdot 4)\end{array}$ & $\begin{array}{l}\text { t002 }(8 \cdot 2) \\
\text { t015 }(6 \cdot 6) \\
\text { t084 }(6 \cdot 6) \\
\text { t008 }(4 \cdot 9) \\
\text { t065 (4.9) } \\
\text { t709 (4.9) }\end{array}$ & $\begin{array}{l}\text { t002 }(16 \cdot 7) \\
\text { t1040 }(8 \cdot 3) \\
\text { t160 }(8 \cdot 3) \\
\text { t709 }(8 \cdot 3) \\
\text { t728 }(8 \cdot 3)\end{array}$ & $\begin{array}{l}\mathrm{t} 160 \dagger, \mathrm{t} 002, \mathrm{t} 008, \\
\mathrm{t} 015, \mathrm{t} 164, \mathrm{t} 505, \\
\mathrm{t} 9208\end{array}$ & & \\
\hline
\end{tabular}

* $S$. aureus detected in one or more of the body sites.

$\dagger$ spa type 160 was found in two individuals, the rest in one each.

\$ The proportion of individuals carrying the spa type at the given body site.

isolates and the occurrence of spa types specified by individual characteristics and geographical region.

\section{RESULTS}

\section{Prevalence}

On the first sampling occasion 179/290 individuals were sampled from nares, throat and groin. From the remaining 111 subjects only samples from one or two of the body sites were available. There were no differences in age, gender and length of stay in the nursing home between individuals sampled on three body sites compared to those sampled from one or two body sites. Overall, 185 isolates of $S$. aureus from 747 samples were obtained. Table 1 shows that the highest prevalence was found in throat samples $(34 \%)$ followed by anterior nares $(31 \%)$, and by combining nares and throat samples the prevalence increased to $48 \%$. There was no significant difference in gender $(P=0.52)$ or mean age $(P=0.14)$ between individuals colonized and not colonized.

In the longitudinal study the 290 individuals were sampled on various occasions $(n=1-5)$ for up to 2 years, due to the open cohort design; $28 \%$ once, $21 \%$ twice, $18 \%$ three times, $28 \%$ four times and $5 \%$ five times. A total of 1860 samples was obtained; nares $(n=739)$, throat $(n=355)$, groin $(n=665)$ and skin lesions $(n=101)$ and $S$. aureus was isolated from 466 of these cultures: nares $(n=240)$, throat $(n=110)$, groin $(n=63)$ and skin lesions $(n=53)$. The accumulated proportion of individuals from whom $S$. aureus could be isolated, increased from $31 \%$ to $60 \%$ and

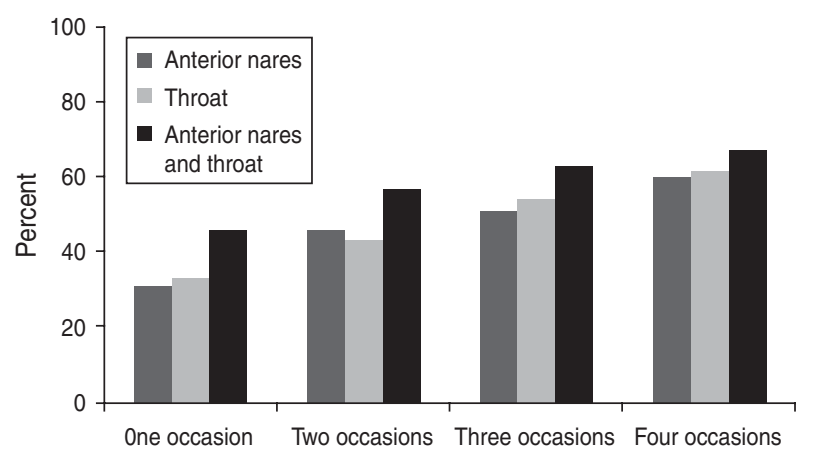

Fig. 1. The prevalence of $S$. aureus colonization rates obtained by the accumulation of results from increasing sampling occasions.

from $34 \%$ to $62 \%$ in nares and throat, respectively, by increasing the number of sampling occasions $(n=4)$, and by combining results from nares and throat the proportion increased to $67 \%$ (Fig. 1).

\section{spa type distribution}

Of the 185 isolates from the first sampling occasion 73 different spa types were identified; 47 (64\%) were only found in single individuals, $14(19 \%)$ in two, and $13(18 \%)$ in three or more individuals. One isolate was non-typable. The most common spa types were t002 (8.3\%), t160 (5.8\%), t015 (5.0\%), t084 (5.0\%) and t705 (3.3\%). No difference in distribution of common spa types was seen between the nares and throat samples (Table 1). All isolates from the groin and skin lesions belonged to spa types also isolated from other body sites. Carriage of two different spa types in one 

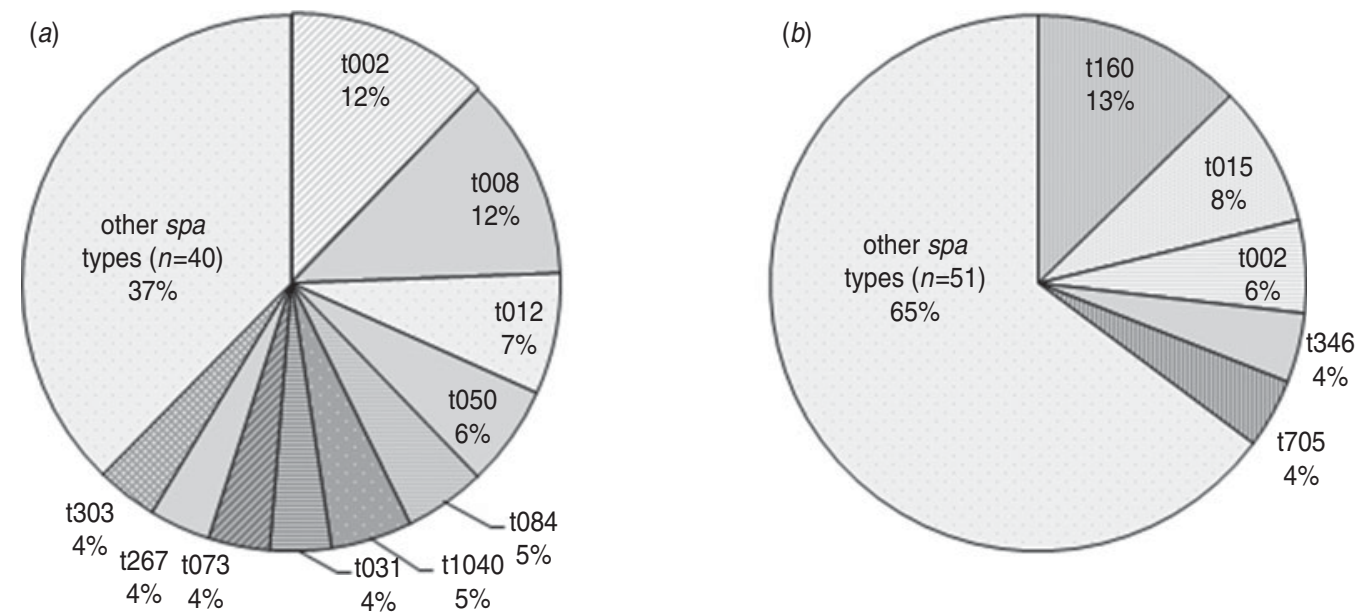

Fig. 2. Geographical distribution of spa types isolated from more than two individuals in the longitudinal study in (a) Eslöv and (b) Jönköping.

individual, was found in 10/43 (23\%) individuals from whom $S$. aureus was isolated from more than one body site on the same occasion.

In the longitudinal study, no temporal differences in the distribution of major spa types were seen (data not shown). Fifty different $s p a$ types distributed over 82 individuals were detected in Eslöv, and in Jönköping 56 different spa types in 71 individuals were found. No spa type was carried by more than two individuals in Linköping. S. aureus of spa type t160 was found in nine individuals in one region, but was not found in the other geographical regions whereas other common spa types were equally distributed (Fig. 2). Seven of the individuals with spa type t160 were living at the same nursing home.

BURP cluster analysis of all spa types $(n=98)$ found in the longitudinal study revealed 10 clusters and 14 singletons (seven spa types shorter than five repeats were excluded from the analysis). The largest cluster was spa-CC 015 including $17 \%$ of all spa types followed by spa-CC 012 (15\% of all spa types), spa-CC 084 ( $9 \%$ of all spa types) and spa-CC 005 (4\% of all spa types). Of the 35 individuals that carried different spa types at any time during the study period, 21 carried the different strains at the same occasion and 18 changed spa type over time. Three individuals carried spa types closely related and in two of these individuals both spa types were present on the same sampling occasion.

\section{Carriage of $S$. aureus over time}

Carriage over time was evaluated in individuals sampled on three occasions (every 6 months) for
1 year. S. aureus was isolated from nares in $20 \%$ of the individuals, and $17 \%$ carried strains of the same spa types over the sampling period (Table 2). Similarly, $7 \%$ of the individuals exhibited throat carriage and all carried the same spa type. The proportion of individuals from whom $S$. aureus was never isolated was $49 \%$ for nares and $46 \%$ for throat samples. There was no significant difference in gender $(P=0 \cdot 40)$, mean age $(P=0.40)$, or length of stay $(P=0.69)$ between those that always carried $S$. aureus and others. Carriage rates based on two sampling occasions within 6 months from nares $(n=216)$ showed $S$. aureus was present in $22 \%$ of the individuals, and $19 \%$ carried strains of the same spa type. S. aureus was not detected in $55 \%$ of the individuals sampled twice from the nares.

\section{Antibiotic susceptibility testing}

From the 290 individuals in the longitudinal study 338 isolates were available for antibiotic susceptibility testing. Of these 44 isolates from 26 (14 male, 12 female) individuals all but one were resistant to a single antibiotic and the other to two antibiotics (ciprofloxacin and fusidic acid). Overall, 18\% of the colonized individuals carried an antibiotic-resistant isolate. None of the isolates was resistant to vancomycin, gentamicin, erythromycin, linezolid or rifampicin and no MRSA was detected. No specific spa type was associated with resistance. The proportion of individuals with resistant isolates was higher in the individuals colonized with two different strains compared to carriers with a single clone $(P=0 \cdot 04)$. Five individuals who carried two different strains were 
Table 2. Repeated detection of $\mathrm{S}$. aureus in individuals sampled on three occasions during one year

\begin{tabular}{|c|c|c|c|c|c|c|}
\hline & \multicolumn{2}{|c|}{ Anterior nares $(n=144)$} & \multicolumn{2}{|c|}{ Throat $(n=46)$} & \multicolumn{2}{|c|}{$\begin{array}{l}\text { Any of three sampling sites } \\
(n=44)^{*}\end{array}$} \\
\hline & $\begin{array}{l}\text { Number of } \\
\text { individuals } \\
(\%)\end{array}$ & $\begin{array}{l}\text { Individuals } \\
\text { with the same } \\
\text { spa type } n(\%)\end{array}$ & $\begin{array}{l}\text { Number of } \\
\text { individuals } \\
(\%)\end{array}$ & $\begin{array}{l}\text { Individuals } \\
\text { with the same } \\
\text { spa type } n(\%)\end{array}$ & $\begin{array}{l}\text { Number of } \\
\text { individuals } \\
(\%)\end{array}$ & $\begin{array}{l}\text { Individuals } \\
\text { with the same } \\
\text { spa type } n(\%)\end{array}$ \\
\hline $\begin{array}{l}\text { S. aureus detected on all } \\
\text { occasions }\end{array}$ & $29(20)$ & $24(17)$ & $3(7)$ & $3(7)$ & $11(25)$ & $9(20)$ \\
\hline $\begin{array}{l}\text { S. aureus detected on two } \\
\text { occasions }\end{array}$ & $15(10)$ & $11(8)$ & $8(17)$ & $6(30)$ & $9(20)$ & $6(14)$ \\
\hline $\begin{array}{l}\text { S. aureus detected on one } \\
\text { occasion }\end{array}$ & $29(20)$ & & $14(30)$ & & $11(25)$ & \\
\hline S. aureus not detected & $71(49)$ & & $21(46)$ & & $13(30)$ & \\
\hline
\end{tabular}

* Individuals sampled from nares, throat and groin on three occasions.

simultaneously colonized with resistant and nonresistant strains.

\section{DISCUSSION}

In this study we investigated the molecular epidemiology of $S$. aureus in a population of the elderly living in nine nursing homes in the south of Sweden. We found a diverse distribution of spa types as well as a local spread of one spa type. As much as $23 \%$ of individuals were colonized simultaneously with isolates of two different spa types and in this group antimicrobial resistance was twice as common as for those colonized with a single strain, and five individuals simultaneously carried resistant and susceptible strains. Furthermore, we show that inclusion of throat sampling with nasal swabs as well as repeated sampling increases the sensitivity of $S$. aureus detection in screening programmes.

The high diversity and the most frequently identified spa types in our study are in agreement with previous data from general populations as well as from hospital settings $[1,2,5-7,29]$. The four most common spa types were also the most prevalent in the Swedish HITS study, which was conducted in the regions of Jönköping and Linköping, but the fifth most common spa type was restricted to the region of Eslöv. This indicates a comparable distribution of spa types between regions and populations and suggests that some strains are more successful colonizers than others, as exemplified by the finding of spa type t160 in a single nursing home. There are conflicting results in the literature on spa-type distribution regarding age and gender $[2,13]$ but we found no differences in these parameters and body site sampled in our study population.

BURP clustering analysis revealed that only $3 / 35$ individuals carrying different spa types at any time were carriers of closely related strains and that almost half of all spa types fell into the four largest clusters. The high rate $(23 \%)$ of individuals harbouring simultaneously two strain types in two or more body sites on a single sampling occasion is noteworthy as only one isolate from each site was subjected to spa typing. A previous study calculated the rate of individuals carrying two different strains of $S$. aureus to be below $7 \%$, based on typing of three colonies from the anterior nares [30]. More recently a study in children reported a similar rate $(26 \%)$ to that found here when 4-15 colonies were typed [31]. The issue of simultaneous carriage of more than one strain is of clinical importance as a more resistant $S$. aureus strain may remain undetected impairing antibiotic treatment, a situation underlined by the recovery of resistant and susceptible phenotypes of different spa types from the same individual, albeit from different body sites. Further studies are required to elucidate the clinical significance of these findings. Furthermore, in epidemiological and transmission studies undetected carriage of multiple strains could lead to misinterpretation of data. However, analyses of dual colonization with different strains based on selecting several colonies from agar plates would be laborious and simplified methods are needed [32].

The highest prevalence of $S$. aureus carriage was found in the throat (34\%), which is in accordance with a previous Swedish study [13]; the nasal colonization rate of $31 \%$ is also comparably high, but in 
agreement with previous results from a Swedish population [1]. Screening protocols for S. aureus carriage based on nares sampling only may underestimate colonization rates. We were able to increase the recovery rate from $31 \%$ to $48 \%$ by combining the culture results obtained from the nares and the throat on one sampling occasion which has also been shown by other studies [20-22]. In addition, repeated sampling occasions further increased the prevalence figures. Thus the use of a sensitive strategy of multiple bodysite sampling on different occasions maximized the estimates of the prevalence of $S$. aureus colonization in this elderly population.

The issue of persistent carriage of $S$. aureus is important, since persistent nasal carriers appear to have an increased risk for endogenous infection [15-17]. There is no general consensus on the definition of persistent carriage [14, 18], and reported frequencies from nasal samples vary between $12 \%$ and $30 \%$ [12, 13, 17, 33-35]. We found $20 \%$ of carriers were repeatedly colonized in the nares on three sampling occasions over 1 year and this rose to $22 \%$ if the result was based on two sampling occasions. The importance of persistent throat carriage, as a risk for endogenous infections and in transmission to others has not been fully evaluated. Recently, a persistent throat carrier rate of $46 \%$ was reported using enrichment broth prior to plating [13]. These are higher figures than the rates of $24 \%$ and $7 \%$ repeatedly colonized in the throat on two and three occasions, respectively, found in our study. However, we only succeeded in obtaining three throat samples from 46 individuals due to sampling difficulties.

When persistent carriage is to be defined, the type identity of the strain of $S$. aureus should be considered [13]. It is not known if the risk inherent in being a persistent carrier is dependent on continuously carrying the same strain. We found that $17 \%$ of the elderly were colonized in the nares with $S$. aureus of the same spa type on all three occasions compared to $20 \%$ for all $S$. aureus strain types and the corresponding figures for two sampling occasions were 19\% and $22 \%$, respectively.

S. aureus was never detected in almost half of nasal $(49 \%)$ and throat $(47 \%)$ swabs of participants and they could thus be regarded as non-carriers, which is in agreement with other findings [12, 14, 17, 33-35]. The basis for the resistance of individuals to colonization is not known, but some spa types seem to be more successful colonizers of humans [36], and little is known about bacterial factors of importance for specific strains to become virulent [37].
In conclusion, we found a high prevalence of $S$. aureus in this elderly population and a local accumulation of one strain was noted in one nursing home. Antibiotic resistance was rare and no MRSA was found, but in individuals colonized with two different strains resistance was higher and simultaneous detection of resistant and non-resistant strains was registered.

\section{ACKNOWLEDGEMENTS}

Financial support was provided by Futurum-the academy of Healthcare, County Council of Jönköping, Sweden, the Medical Research Council of SouthEast Sweden (FORSS) and the County Council of Östergötland, Sweden.

We acknowledge the clinical microbiology laboratory in Halmstad for providing S. aureus isolates from part of the study.

\section{DECLARATION OF INTEREST}

None.

\section{REFERENCES}

1. den Heijer CD, et al. Prevalence and resistance of commensal Staphylococcus aureus, including meticillinresistant $S$. aureus, in nine European countries: a crosssectional study. Lancet Infectious Diseases 2013; 13: 409-415.

2. Sangvik M, et al. Age- and gender-associated Staphylococcus aureus spa types found among nasal carriers in a general population: the Tromso Staph and Skin Study. Journal of Clinical Microbiology 2011; 49: 4213-4218.

3. Mostofsky E, Lipsitch M, Regev-Yochay G. Is methicillin-resistant Staphylococcus aureus replacing methicillin-susceptible $S$. aureus? Journal of Antimicrobial Chemotherapy 2011; 66: 2199-2214.

4. David MZ, et al. Methicillin-susceptible Staphylococcus aureus as a predominantly healthcare-associated pathogen: a possible reversal of roles? PloS One 2011; 6: e18217.

5. Ghasemzadeh-Moghaddam H, et al. Methicillin-susceptible Staphylococcus aureus from clinical and community sources are genetically diverse. International Journal of Medical Microbiology 2011; 301: 347-353.

6. Miko BA, et al. Molecular characterization of methicillin-susceptible Staphylococcus aureus clinical isolates in the United States, 2004 to 2010. Journal of Clinical Microbiology 2013; 51: 874-879.

7. Grundmann H, et al. Geographic distribution of Staphylococcus aureus causing invasive infections in Europe: a molecular-epidemiological analysis. PLoS Medicine 2010; 7: e1000215.

8. Goering RV, et al. Molecular epidemiology of methicillin-resistant and methicillin-susceptible Staphylococcus 
aureus isolates from global clinical trials. Journal of Clinical Microbiology 2008; 46: 2842-2847.

9. Bonomo RA. Multiple antibiotic-resistant bacteria in long-term-care facilities: an emerging problem in the practice of infectious diseases. Clinical Infectious Diseases 2000; 31: 1414-1422.

10. Pfingsten-Wurzburg S, et al. Prevalence and molecular epidemiology of meticillin-resistant Staphylococcus aureus in nursing home residents in northern Germany. Journal of Hospital Infection 2011; 78: 108-112.

11. Denis O, et al. Epidemiology of methicillin-resistant Staphylococcus aureus (MRSA) among residents of nursing homes in Belgium. Journal of Antimicrobial Chemotherapy 2009; 64: 1299-1306.

12. Kluytmans J, van Belkum A, Verbrugh H. Nasal carriage of Staphylococcus aureus: epidemiology, underlying mechanisms, and associated risks. Clinical Microbiology Reviews 1997; 10: 505-520.

13. Nilsson P, Ripa T. Staphylococcus aureus throat colonization is more frequent than colonization in the anterior nares. Journal of Clinical Microbiology 2006; 44: 3334 3339.

14. Wertheim HF, et al. The role of nasal carriage in Staphylococcus aureus infections. Lancet Infectious Diseases 2005; 5: 751-762.

15. Wertheim HF, et al. Risk and outcome of nosocomial Staphylococcus aureus bacteraemia in nasal carriers versus non-carriers. Lancet 2004; 364: 703-705.

16. von Eiff C, et al. Nasal carriage as a source of Staphylococcus aureus bacteremia. Study Group. New England Journal of Medicine 2001; 344: 11-16.

17. van Belkum A, et al. Reclassification of Staphylococcus aureus nasal carriage types. Journal of Infectious Diseases 2009; 199: 1820-1826.

18. Verhoeven PO, et al. An algorithm based on one or two nasal samples is accurate to identify persistent nasal carriers of Staphylococcus aureus. Clinical Microbiology and Infection 2012; 18: 551-557.

19. Mertz D, et al. Throat swabs are necessary to reliably detect carriers of Staphylococcus aureus. Clinical Infectious Diseases : 2007; 45: 475-477.

20. Smith TC, et al. Molecular and epidemiologic predictors of Staphylococcus aureus colonization site in a population with limited nosocomial exposure. American Journal of Infection Control 2012; 40: 992-996.

21. Mernelius S, et al. The role of broth enrichment in Staphylococcus aureus cultivation and transmission from the throat to newborn infants: results from the Swedish hygiene intervention and transmission of S. aureus study. European Journal of Clinical Microbiology \& Infectious Diseases. Published online: 2 July 2013. doi:10.1007/s10096-013-1917-6.

22. Andrews JI, et al. Screening for Staphylococcus aureus carriage in pregnancy: usefulness of novel sampling and culture strategies. American Journal of Obstetrics and Gynecology 2009; 201: 396 e1-5.

23. Olofsson M, et al. Colonization with Staphylococcus aureus in Swedish nursing homes: a cross-sectional study. Scandinavian Journal of Infectious Diseases 2012; 44: $3-8$.
24. Ernsth Bravell M, et al. How to assess frailty and the need for care? Report from the Study of Health and Drugs in the Elderly (SHADES) in community dwellings in Sweden. Archives of Gerontology and Geriatrics 2011; 53: 40-45.

25. Nilsson $\mathbf{P}$, Alexandersson H, Ripa T. Use of broth enrichment and real-time PCR to exclude the presence of methicillin-resistant Staphylococcus aureus in clinical samples: a sensitive screening approach. Clinical Microbiology and Infection 2005; 11: 1027-1034.

26. Kahl BC, et al. Variation of the polymorphic region $\mathrm{X}$ of the protein A gene during persistent airway infection of cystic fibrosis patients reflects two independent mechanisms of genetic change in Staphylococcus aureus. Journal of Clinical Microbiology 2005; 43: 502-505.

27. Harmsen D, et al. Typing of methicillin-resistant Staphylococcus aureus in a university hospital setting by using novel software for spa repeat determination and database management. Journal of Clinical Microbiology 2003; 41: 5442-5448.

28. Mellmann A, et al. Based Upon Repeat Pattern (BURP): an algorithm to characterize the longterm evolution of Staphylococcus aureus populations based on spa polymorphisms. BMC Microbiology 2007; 7: 98 .

29. Mernelius S, et al. The effect of improved compliance with hygiene guidelines on transmission of Staphylococcus aureus to newborn infants: The Swedish Hygiene Intervention and Transmission of $S$ aureus study. American Journal of Infection Control 2013; 41: 585-590.

30. Cespedes C, et al. The clonality of Staphylococcus aureus nasal carriage. Journal of Infectious Diseases 2005; 191: 444-452.

31. Mongkolrattanothai $\mathbf{K}$, et al. Simultaneous carriage of multiple genotypes of Staphylococcus aureus in children. Journal of Medical Microbiology 2011; 60: 317-322.

32. Matussek A, et al. Analyzing multiclonality of Staphylococcus aureus in clinical diagnostics using spa-based denaturing gradient gel electrophoresis. Journal of Clinical Microbiology 2011; 49: 3647-3648.

33. Eriksen NH, et al. Carriage of Staphylococcus aureus among 104 healthy persons during a 19-month period. Epidemiology and Infection 1995; 115: 51-60.

34. Hu L, et al. Typing of Staphylococcus aureus colonising human nasal carriers by pulsed-field gel electrophoresis. Journal of Medical Microbiology 1995; 42: 127-132.

35. Nouwen JL, et al. Predicting the Staphylococcus aureus nasal carrier state: derivation and validation of a 'culture rule'. Clinical Infectious Diseases 2004; 39: 806811.

36. Melles DC, et al. Overlapping population structures of nasal isolates of Staphylococcus aureus from healthy Dutch and American individuals. Journal of Clinical Microbiology 2008; 46: 235-241.

37. Stark L, et al. Staphylococcus aureus isolates from blood and anterior nares induce similar innate immune responses in endothelial cells. APMIS 2009; 117: 814 824. 